\title{
An Improved Model for Bending of Thin Viscoelastic Plate on Elastic Foundation
}

\author{
Zhi-Da $\mathrm{Li}^{1,2}$, Ting-Qing Yang ${ }^{1}$, Wen-Bo Luo ${ }^{3}$ \\ ${ }^{1}$ Department of Mechanics, Huazhong University of Science and Technology, Wuhan, China; zhidali@163.com \\ ${ }^{2}$ School of transportation, Wuhan University of Technology, Wuhan, China \\ ${ }^{3}$ College of Civil Engineering and Mechanics, Xiangtan University, Xiangtan, China
}

Received 10 June 2009; revised 16 July 2009; accepted 20 July 2009.

\begin{abstract}
An improved model for bending of thin viscoelastic plate resting on Winkler foundation is presented. The thin plate is linear viscoelastic and subjected to normal distributed loading, the effect of normal stress along the plate thickness on the deflection and internal forces is taken into account. The basic equations for internal forces and stress distribution are derived based on the general viscoelastic theory under small deformation condition. The reduced equations for elastic case are given as well. It is shown that the proposed model reveals a larger flexural rigidity compared to that in classic models, in which the normal stress along the plate thickness is neglected.
\end{abstract}

Keywords: Thin Viscoelastic Plate; Deformable Foundation; Flexural Rigidity; Winkler Foundation

\section{INTRODUCTION}

The analysis of soil-structure interaction has a wide range of applications in structural and geotechnical engineering, for instance, in highway asphalt pavement engineering, the pavement is usually treated as thin elastic/viscoelastic plate structure resting on elastic/viscoelastic foundation. Due to the complexity of the actual behavior of foundations, many idealized foundation models have appeared in the literature [1]. The simplest of those models, which was proposed in 1867 by Winkler, assumes that the soil medium consists of a system of mutually independent spring elements. There are many papers dealing with the elastic beam or plates resting on the Winkler foundation in the literature [2,3]. As computational power has developed, more realistic modeling of soil-structure interaction has become possible. Because of the importance of viscoelastic nature of the ma- terials used for structures, e.g. asphalt layer of pavement structure, many works have been done to deal with the bending behaviour of thin viscoelastic plate on elastic/viscoelastic foundation. Most of such works utilized the models similar to those for bending of elastic plate. Mase [4] directly offered the fundamental equations for bending of viscoelastic plate by replacing the flexural rigidity of elastic plate with the rigidity of viscoelastic plate. Radovskii [5] discussed the problem of treating highway and airport pavement as thin viscoelastic plate. Pister [6], Robertson [7] and Hewitt and Mazumdar [8] applied the elasticity-viscoelasticity correspondence principle to get the solution of the bending problem of viscoelastic plate. In contrast to dealing with the viscoelastic plate on elastic foundation, some attempts have also been made to solve the bending problem of elastic plate resting on viscoelastic foundation. Sonoda et al $[9,10]$ studied the circular and rectangular plate on linear viscoelastic foundation. Lin [11] and Yang et al. [12] analyzed the dynamic response of circular plate resting on viscoelastic half space. All of the above studies followed the classic model and traditional flexural rigidity for thin plate bending, in which the Kirchhoff hypothesis was used and $\sigma_{z z}$ was neglected [13]. However, in the case of large lateral load subjecting to thin plate resting on a deformable foundation with relatively large rigidity, the bearing stresses along the plate thickness, $\sigma_{z z}$, may not be ignored. Furthermore, it is the bearing stress of the plate that transfer the active lateral load to the foundation, the boundary condition on the main surfaces of the plate should be satisfied. Therefore it is necessary to develop a method to consider the effect of lateral normal stress. In this paper, we first seek to develop a modified Kirchhoff theory for thin viscoelastic plate resting on Winkler foundation, in which the effect of the lateral normal stress is considered. Then we reduce the obtained results to the problem of thin elastic plate resting on elastic foundation, and a different elastic flexural rigidity is obtained. 


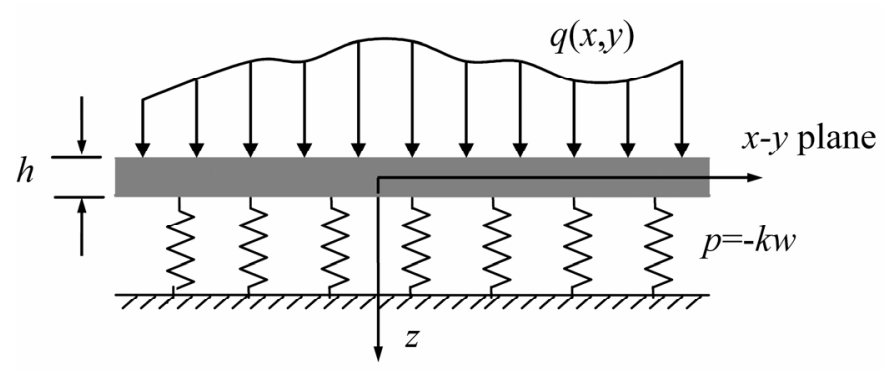

Figure 1. Thin viscoelastic plate resting on a Winkler foundation.

\section{VISCOELASTIC PLATE ON WINKLER FOUNDATION: AN IMPROVED MODEL}

Figure 1 illustrates a thin viscoelastic plate of a thickness $h$, its middle-plane coincides with the $\mathrm{x}-\mathrm{y}$ plane of the reference coordinate system. Let the upper surface $(z=-h / 2)$ be subjected to a normal distributed loading with intensity of $q(x, y)$, while the lower surface $(z=h / 2)$ rests on a Winkler type foundation. In the Winkler foundation model, the foundation for the plate is assumed to act like a set of springs. Thus the foundation reaction force can be written as $p=-k w$, where $k$ denotes the elastic stiffness of the foundation and $w$ is the lateral deflection of the plate.

For a linear viscoelastic plate, the equilibrium equations, the strain-displacement relations and the constitutive equations are given, respectively, by

$$
\begin{gathered}
\sigma_{i j ; j}=0 \\
\varepsilon_{i j}=\frac{1}{2}\left(u_{i ; j}+u_{j ; i}\right) \\
s_{i j}=2 G_{1} * d e_{i j}, \quad \sigma_{k k}=3 K_{1} * d \varepsilon_{j j}
\end{gathered}
$$

where the body forces are neglected, $G_{1}(t)$ and $K_{1}(t)$ are the shear modulus function and volume modulus function, respectively, the $*$ denotes convolution product. The deviatoric components of stress and strain tensors are

$$
s_{i j}=\sigma_{i j}-\frac{1}{3} \delta_{i j} \sigma_{k k} \quad \text { and } \quad e_{i j}=\varepsilon_{i j}-\frac{1}{3} \delta_{i j} \varepsilon_{k k}
$$

For simplicity, the displacement components along $x$, $y$ and $z$ axis are denoted by $u, v$ and $w$ respectively. Assuming $\varepsilon_{z z}=0, \varepsilon_{z x}=0, \varepsilon_{z y}=0$, from Eq.2 we have

$$
w=w(x, y, t), \quad u=-z w_{; x}, \quad v=-z w_{; y}
$$

and

$$
\varepsilon_{x x}=-z w_{; x x}, \quad \varepsilon_{y y}=-z w_{; y y}, \quad \varepsilon_{x y}=-z w_{; x y}, \quad \varepsilon_{k k}=-z \nabla^{2} w
$$

Combining Eq.4 and Eq.3, along with substitution of Eq.6 into it, the stress components can be written as

$$
\begin{gathered}
\sigma_{x x}=-2 z G_{1} * d w_{; x x}-z\left(K_{1}-\frac{2}{3} G_{1}\right) * d\left(\nabla^{2} w\right) \\
\sigma_{y y}=-2 z G_{1} * d w_{; y y}-z\left(K_{1}-\frac{2}{3} G_{1}\right) * d\left(\nabla^{2} w\right) \\
\sigma_{x y}=-2 z G_{1} * d w_{; x y}
\end{gathered}
$$

The other three components of the stress tensor can be obtained by using the equilibrium equations, Eq.1. Integrating the first and second representations of Eq.1 over $z$ and considering the boundary condition $\sigma_{z x}=\sigma_{z y}=0$ for $z= \pm h / 2$ (i.e. there is no shear stress on the plate surface and no friction between plate and foundation), we get

$$
\begin{aligned}
& \sigma_{z x}=\left(z^{2}-\frac{h^{2}}{4}\right)\left(\frac{K_{1}}{2}+\frac{2}{3} G_{1}\right) * d\left(\nabla^{2} w_{; x}\right) \\
& \sigma_{z y}=\left(z^{2}-\frac{h^{2}}{4}\right)\left(\frac{K_{1}}{2}+\frac{2}{3} G_{1}\right) * d\left(\nabla^{2} w_{; y}\right)
\end{aligned}
$$

The third representation of Eq.1 is

$$
\frac{\partial \sigma_{z z}}{\partial z}=-\frac{\partial \sigma_{z x}}{\partial x}-\frac{\partial \sigma_{z y}}{\partial y}
$$

Substituting Eqs.10 and $\mathbf{1 1}$ into the above equation and integrating it over $z$ yields

$$
\sigma_{z z}=\left[\frac{h^{2}}{4}\left(z-\frac{h}{2}\right)-\frac{1}{3}\left(z^{3}-\frac{h^{3}}{8}\right)\right]\left(\frac{K_{1}}{2}+\frac{2}{3} G_{1}\right) * d\left(\nabla^{4} w\right)-k w
$$

It should be noted here that the stress boundary condition $\sigma_{z z}=-k w$ at the lower surface $(z=h / 2)$ of the plate is used to determine the integral constant. Moreover, $\sigma_{z z}=-q$ at the upper surface $(z=-h / 2)$ of the plate, thus

$$
\frac{h^{3}}{12}\left(K_{1}+\frac{4}{3} G_{1}\right) * d\left(\nabla^{4} w\right)=q-k w
$$

This is a differential-integral equation interrelating the materials' properties, the applied normal loads and the corresponding lateral deflection.

We denote by $M_{i j}$ the bending moments and the twisting moments, and $Q_{j}$ the shear forces

$$
M_{i j}=\int_{-h / 2}^{h / 2} \sigma_{i j} z d z, \quad Q_{j}=\int_{-h / 2}^{h / 2} \sigma_{z j} d z
$$


Table 1. Variation of $D_{1} / D$ with Poisson ratio.

\begin{tabular}{cccccccccc}
\hline$\mu$ & 0 & 0.2 & 0.25 & 0.30 & 0.35 & 0.40 & 0.45 & 0.49 & 0.5 \\
\hline$D_{1} / D$ & 1 & 1.067 & 1.125 & 1.225 & 1.408 & 1.80 & 3.025 & 13.005 & $\infty$ \\
\hline
\end{tabular}

Substituting Eqs.7-11 into the above expressions, we get

$$
\begin{gathered}
M_{x x}=-\frac{h^{3}}{12}\left[2 G_{1} * d w_{; x x}+\left(K_{1}-\frac{2}{3} G_{1}\right) * d\left(\nabla^{2} w\right)\right] \\
M_{y y}=-\frac{h^{3}}{12}\left[2 G_{1} * d w_{; y y}+\left(K_{1}-\frac{2}{3} G_{1}\right) * d\left(\nabla^{2} w\right)\right] \\
M_{x y}=-\frac{h^{3}}{6} G_{1} * d w_{; x y} \\
Q_{x}=-\frac{h^{3}}{12}\left(K_{1}+\frac{4}{3} G_{1}\right) * d\left(\nabla^{2} w_{; x}\right) \\
Q_{y}=-\frac{h^{3}}{12}\left(K_{1}+\frac{4}{3} G_{1}\right) * d\left(\nabla^{2} w_{; y}\right)
\end{gathered}
$$

Furthermore, the stress components can be expressed in the form of $M_{i j}$ and $Q_{j}$ as follows:

$$
\begin{gathered}
\sigma_{i j}=\frac{12}{h^{3}} z M_{i j}, \quad(i, j=x, y) \\
\sigma_{z j}=\frac{3}{2 h}\left[1-\left(\frac{2 z}{h}\right)^{2}\right] Q_{j}, \quad(j=x, y) \\
\sigma_{z z}=-2(q-k w)\left(\frac{z}{h}-\frac{1}{2}\right)^{2}\left(1+\frac{z}{h}\right)-k w
\end{gathered}
$$

The forms of Eqs.19-21 are as same as those for elastic bending plate $[13,14]$, however it should be noted that $M_{i j}$ and $Q_{j}$ involved are the moments and the shear forces in viscoelastic cases.

\section{ELASTIC PLATE ON WINKLER FOUNDATION}

In the case of thin elastic plate with material constants of $G$ and $K$, resting on a Winkler foundation, the Eq.13 reduces to the following equation

$$
D_{1} \nabla^{4} w=q-k w
$$

in which

$$
D_{1}=\frac{h^{3}}{12}\left(K+\frac{4}{3} G\right)=\frac{E h^{3}}{12\left(1-\mu^{2}\right)} \cdot \frac{(1-\mu)^{2}}{1-2 \mu}
$$

$E$ and $\mu$ are the elastic modulus and Poisson ratio of the elastic plate, respectively.

Again, it can be seen that the form of the control equation for elastic plate bending is similar to that derived from classic theory. However the flexural rigidity $D_{1}$ is different from that in classic theory, which gives $D=E h^{3} / 12\left(1-\mu^{2}\right)$. Obviously, $D_{1} / D=\left(1-\mu^{2}\right) /(1-2 \mu)$. It can be seen that $D_{1} / D$ increases with the Poisson's ratio. Several results are listed in Table 1. For most engineering structural materials, $\mu$ is about 0.3 [13], thus the flexural rigidity given in the present model is about $22 \%$ larger than that in the classic model.

In elastic cases, Eqs.14-18 reduces to the followings in terms of $D_{1}$ :

$$
\begin{gathered}
M_{x x}=-D_{1}\left[w_{; x x}+\frac{\mu}{1-\mu} w_{; y y}\right] \\
M_{y y}=-D_{1}\left[w_{; y y}+\frac{\mu}{1-\mu} w_{; x x}\right] \\
M_{x y}=-D_{1}\left[\frac{1-2 \mu}{1-\mu}\right] w_{; x y} \\
Q_{x}=-D_{1} \nabla^{2} w_{; x} \\
Q_{y}=-D_{1} \nabla^{2} w_{; y}
\end{gathered}
$$

Moreover, the stress distribution in the elastic plate can be read in the same forms as Eqs.19-21 if the moments and shear forces are given by Eqs.24-28.

\section{CONCLUDING REMARKS}

We have derived in this paper the basic equations for bending of viscoelastic thin plate on Winkler foundation. In classic treatment, the normal stress $\sigma_{z z}$ is assumed to be zero, which is the main characteristic of the plane stress state in classic elastic theory, and thus the corresponding classic bending model of thin elastic plate can be considered as a plane stress model. In contrast with the classic treatment, in the proposed model in this paper, $\sigma_{z z}$ is taken into account and the general viscoelastic constitutive equation is used to represent the thin plate behavior, and the plate thickness is further set to be constant, i.e. $\varepsilon_{z z}=0$, which is the main characteristic of the plane strain state in classic elastic theory. Thus we may consider this improved model as a quasi-plane strain model.

\section{ACKNOWLEDGMENTS}

The study was supported by the National Natural Sci- 
ence Foundation of China (No.10372074).

\section{REFERENCES}

[1] Selvadurai, P.S. (1979) Elastic analysis of soil-foundation interaction. Amsterdam: Elsevier.

[2] Kaschiev, M.S. and Mikhajlov, K. (1995) A beam resting on a tensionless Winkler foundation. Computer and Structures, 55, 261-264.

[3] Kim, S.M. and Roesset J.M. (1998) Moving loads on a plate on elastic foundation. Journal of Engineering Mechanics, 124, 1010-1017.

[4] Mase, G.E. (1960) Behavior of viscoelastic plates in bending. Journal of Engineering Mechanics, 86, 25-39.

[5] Radovskii, S. (1980) Application of the calculation scheme for a layered viscoelastic medium to the estimation of the stressed state of highways and airport pavements with moving loads. Soviet Applied Mechanics, 15, 940-946.

[6] Pister, K.S. (1961) Viscoelastic plate on a viscoelastic foundation. Journal of Engineering Mechanics, 87, 43-54.

[7] Robertson, S.R. (1971) Solving the problem of forced motion of viscoelastic plates by Valanis' method with an application to a circular plate. Journal of Sound and $\mathrm{Vi}$ bration, 14, 263-278.

[8] Hewitt, J.S. and Mazumdar, J. (1974) Vibration of viscoelastic plates under transverse load by the method of constant deflection contours. Journal of Sound and Vibration, 33, 319-333.

[9] Sonoda, K., Ishio, T. and Kobayashi, H. (1978) Circular plates on linear viscoelastic foundations. Journal of Engineering Mechanics, 104, 819-828.

[10] Sonoda, K. and Kobayashi, H. (1980) Rectangular plates on linear viscoelastic foundations. Journal of Engineering Mechanics, 106, 323-338.

[11] Lin, Y.J. (1978) Dynamic response of circular plates resting on viscoelastic half space. Journal of Applied Mechanics, 45, 379-384.

[12] Yang, T.Q., Wang, R. and Yang, Z.W. (1991) Dynamic response of a viscoelastic circular plate on a viscoelastic half space foundation. In: Zyczkowski $M$ (ed.) Creep in Structures, Berlin: Springer-Verlag, 685-692.

[13] Timoshenko, S. and Woinowsky-Krieger, S. (1959) Theory of Plates and Shells. New York: McGraw-Hill.

[14] Yang, T.Q., Lu, H.B. and Huang, Y.Y. (1987) Quasi-static bending of a thin viscoelastic plate on foundations. Journal of Huazhong University of Science and Technology, 15, 1-6 (in Chinese). 